\title{
MSTIDs impact on GNSS observations and its mitigation in rapid static positioning at medium baselines
}

\author{
Rafał Sieradzki, Jacek Paziewski
}

University of Warmia and Mazury in Olsztyn, Poland

\author{
Article history \\ Received October 16, 2015; accepted December 9, 2015. \\ Subject classification: \\ Precise satellite positioning, GNSS, Ionosphere, MSTID.
}

\section{ABSTRACT}

Total electron content (TEC) fluctuation in the ionosphere is one of the main unresolved problems degrading ambiguity resolution and thus affect the reliability of satellite positioning. With regard to conditions prevailing at mid-latitudes, the impact of the upper atmospheric layers is primarily associated with the occurrence of medium-scale traveling ionospheric disturbances (MSTIDs). This study contains the combined analysis involving MSTIDs patterns observed in raw phase data and their impact on rapid static positioning. The first part is aimed at discrepancies in wavelike MSTIDs signatures detected in time series of high elevated measurements. It was demonstrated that the differences in slant ionospheric delays at medium baselines often exceed 0.5 TEC units and more importantly, the detected MSTIDs patterns were highly mutable. The next section presents the impact of MSTIDs on double differenced ionospheric delays and performance of rapid static positioning. The positioning was executed using two approaches. The first one adopted as a benchmark strategy was the geometry-based relative positioning model with weighted ionosphere and troposphere parametrization. The second approach was the improved version of the former. It used the rate of TEC corrections, which allowed the mitigation of MSTIDs through the reduction of epoch-wise ionospheric delays to one parameter for each double-differenced arc. The comparison of results for both strategies indicated significant improvement of positioning performance after the application of proposed algorithm.

\section{Introduction}

Nowadays Global Navigation Satellite Systems (GNSS) measurements are applied in a broad spectrum of geodetic and geoscientific activities, e.q. surveying, navigation, GNSS seismology and meteorology [Zumberge et al. 1997, Li et al. 2013, Dawidowicz and Krzan 2014, Gioia et al. 2015a, Li et al. 2015a, Wilgan et al. 2015]. A commonly used approach in geodetic applications is the rapid static mode. In this approach the positioning relies on simultaneously processed dual-frequency observations and a geometry-based model. One of the highly effective models based on geometry is the iono-

sphere weighted model [Teunissen 1997, Hu et al. 2005, Cellmer et al. 2013, Paziewski and Wielgosz 2014, Paziewski 2015b]. The reliability of this method is strongly related to the impact of the ionosphere, which increases correspondingly to the baseline length and thus deteriorates ambiguity fixing feasibility. Thanks to recent developments in the field of GNSS algorithms involving the application of network atmospheric corrections and multi GNSS signals the practicable baseline length has extended to a few dozen kilometers [Odijk 2000, Li et al. 2014, Odolinski et al. 2015, Paziewski et al. 2015]. A more reliable network positioning is related to the accuracy of interpolation of ionospheric delays. In this case, the pre- or simultaneous processing of data from reference stations allows the retrieval of information on total electron content (TEC) which can be subsequently applied to obtain the interpolated values of ionospheric delays at rover position. Consequently the reliable GNSS positioning even in a single epoch regime is usually feasible. The second possible way of ionospheric impact mitigation in real-time measurements is the application of predicted GNSS-based TEC maps [Wielgosz et al. 2008, Garcia-Rigo et al. 2011]. However, the efficiency of this strategy depends on many factors such as spatial and temporal resolution of maps, site position and predominantly the occurrence of different-scale disturbances of electron content.

The introduced above boundary value of the distance between reference station(s) and rover corresponds to quiet ionosphere. In this case, TEC changes observed in all domains (latitude, longitude, time) are generally smoothed, which enables precise GNSS positioning. However, the distribution of TEC in the upper part of the atmosphere can be strongly distorted by the different-scale ionospheric irregularities. Their occurrence leads to high slant TEC (STEC) discrepancies observed even at short and medium baselines. The possible scale 
of this effect depends on many factors e.g. geomagnetic latitude and local time, but generally the most frequent and intense ionospheric disturbances occur at equatorial and high latitudes [Spencer and Mitchell 2007, Ngwira et al. 2013, Sieradzki 2015]. The fluctuations of TEC observed at these regions can reach up to a few TEC units (TECU) in a one-minute interval, making precise GNSS positioning extremely difficult [Jacobsen and Schäfer 2012, Sieradzki and Paziewski 2015]. It should be mentioned that the violent Sun-Earth connection events (e.g. solar flares, solar radio emissions, coronal mass ejections) may strongly affect GNSS positioning at entire globe. The consequence of the aforementioned solar phenomena is an increased ionospheric activity (forming of disturbances, high gradients and anomalies in the electron content distribution) and more frequent occurrence of cycle-slips as well [Jin et al. 2008, Prikryl et al. 2014, Gioia et al. 2015b].

In relation to the mid-latitudes, the most common perturbations of ionospheric plasma are medium-scale travelling ionospheric disturbances (MSTIDs). Regardless of a low amplitude of TEC fluctuations associated with these irregularities, they may also significantly distort the reliability of relative GNSS positioning [HernándezPajares et al. 2006a, Crocetto et al. 2008, Lejeune et al. 2012]. Moreover, considering that MSTIDs are frequent and unpredictable in context of their impact on GNSS observations, they represent the challenging problem for precise relative positioning. In order to counteract this effect, two major approaches are applied. The first relies on informing users of increased ionospheric activity through variant indices like I95, Disturbance Ionosphere Index (DIX) and Single Receiver Medium Scale Traveling Ionospheric Disturbances index (SRMTID) [Wanninger 2004, Jakowski et al. 2012a, Hernández-Pajares et al. 2012, respectively]. Although these parameters do not provide any additional information to be used for the mitigation of MSTIDs, they describe the ionospheric conditions and consequently the potential impact of the upper parts of the atmosphere. The second approach is directly oriented on the improvement of GNSS positioning reliability during disturbed ionospheric conditions. With regard to this point, it is worth to mention the algorithm of positioning with weights relied on the values of scintillation parameters [Aquino et al. 2009, Alves da Silva et al. 2010]. Furthermore, the extensive research on MSTID characterization and mitigation of their impact was conducted at the Technical University of Catalonia (UPC). The approach developed at UPC was based on the modelling of MSTID waves using a subnetwork of reference stations [HernándezPajares et al. 2006a, 2006b].

Despite noticeable progress, MSTID impact miti- gation is still one of most crucial problems in precise GNSS positioning. This case study analysis is aimed at the influence of mid-latitude MSTIDs on phase observations and as a result on the performance of rapid GNSS positioning using a geometry-based ionosphere weighted model. Furthermore, the authors present a modification of the aforementioned model based on the rate of TEC (ROT) corrections which enables the reduction of epoch-varying ionospheric parameters to one parameter per each arc.

The paper is organized as follows. The second section focuses on differences in MSTID signatures observed at medium range baselines, which in fact lead to growth in double-differenced ionospheric delays. The following section demonstrates the novel algorithm in detail and comparative analysis of GNSS positioning performance obtained using the standard ionosphere weighted model and its proposed modification. It also introduces the impact of MSTIDs on double-differenced ionospheric delays and its relationship with elevation angle of observations. The last section presents a summary of this contribution.

\section{MSTIDs and their monitoring using GNSS obser- vations}

The travelling ionospheric disturbances (TIDs) occurring at mid-latitudes are identified as plasma density fluctuations featured by wavelike pattern. The literature concerned with this subject usually divides these structures into two classes: large-scale (LSTID) and medium-scale (MSTID) [Shiokawa et al. 2005, Oinats et al. 2015]. Beside the differences in their spatiotemporal characteristics, both kinds of disturbances have a distinct origin. The first of them is thought to be generated at high latitudes as a result of geomagnetic activity and subsequently flows equatorward. In the case of MSTIDs, they seems to be primarily originated by atmospheric gravity waves (AGW) and interaction between neutral and ionized parts of the atmosphere [Jakowski et al. 2012b]. As MSTIDs are usually classified disturbances with sizes $100-250 \mathrm{~km}$, periods from 10 minutes to one hour and velocities $50-300 \mathrm{~m} / \mathrm{s}$. Recent climatological studies have proven the strong seasonal variations in their occurrence [Hernández-Pajares et al. 2012, Otsuka et al. 2013, Wautelet and Warnant 2014]. With GNSS positioning, it is essential for midlatitude MSTIDs to be frequent and unpredictable phenomenons, which deteriorate phase and pseudorange observations. The impact of MSTIDs on double-differenced GNSS observations at analyzed region, can reach up to about $10 \mathrm{ppm}$ of baseline length what correspond to $0.5 \mathrm{~m}$ at $50 \mathrm{~km}$.

Dual-frequency GNSS observations are widely 
known to be also useful in atmospheric research involving the monitoring of the TEC state and its fluctuations associated with different-scale irregularities. The occurrence of MSTIDs at mid-latitudes is one of the effects which can be analyzed using GNSS measurements. Their detection is usually performed with detrended data from a geometry-free combination (Equation 1)

$$
L_{4}=L_{1}-L_{2}=I_{4}+\lambda_{1} N_{1}-\lambda_{2} N_{2}[m]
$$

where:

$L_{1}, L_{2}$ - the carrier phase observations at both frequencies $\lambda_{1}, \lambda_{2}$ - signals wavelengths

$I_{4}$ - slant ionospheric delay related to $L_{4}$ signal $N_{1}, N_{2}$ - integer ambiguities.

Assuming the invariance of the ambiguity term, all changes observed in this combination are related to STEC fluctuations. Nevertheless, due to relatively long time span used for MSTID monitoring (up to a few hour) it is necessary to detect the possible cycle-slips in phase data. In the presented analysis this step of preprocessing was executed using rate of TEC algorithm proposed by Liu [2011] wherein the cycle-slips are found through the comparison of real rate of TEC values and their expected values computed using temporal STEC differences at previous epochs. In our case as a threshold limit of phase slip occurrence was adopted level of $0.3 \mathrm{TECU} / 30 \mathrm{~s}$. If the rate of TEC exceeded this boundary, all following data in STEC time series were shifted by a constant value equal the difference between real rate of TEC and its computed equivalent.

The preprocessed arcs of geometry-free phase data have to be detrended in order to eliminate the longterm effects mostly driven by diurnal variations of electron content and changes of elevation angle. After this step the information on absolute STEC value is lost, but fluctuations of ionospheric conditions are detected only using precise phase measurements. As a result, the signatures of MSTIDs are clearly visible as wavelike patterns in the time series of individual satellite observations. Low-frequency components from geometryfree observations are usually eliminated with high-pass filtering or differencing [Hernández-Pajares et al. 2006b]. In our case, the levelling process of long-term changes is performed using a third-degree polynomial fitting with the minimal values of the STEC time series for each observation arc. In order to perform this step automatically, the fitting is executed in an iterative mode. At first, the polynomial coefficients are determined for entire STEC time series involving observations with elevation angle higher than 30 degree. In the next three iterations only points below the polynomial computed in previous step are processed. In order to

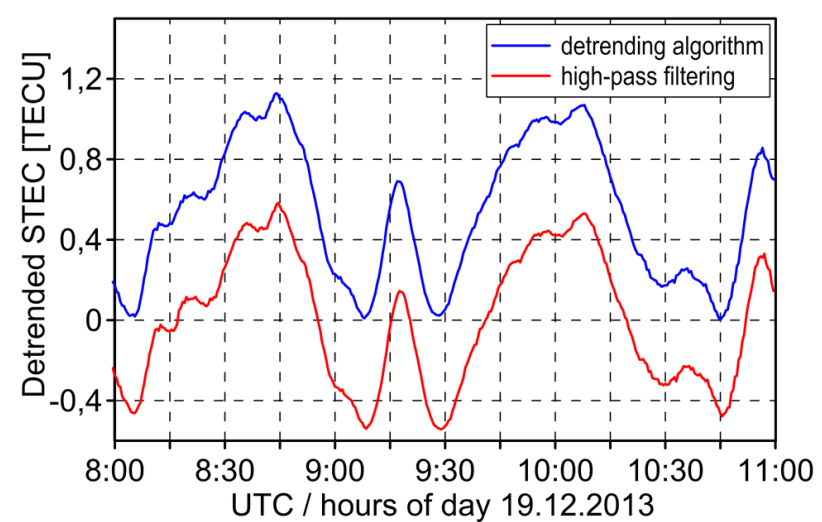

Figure 1. The STEC fluctuations at DOMA station for GPS PRN 19 detrended using (a) fitting method (blue line), (b) high-pass filtering method (red line).

expedite reaching of STEC local minima, the fitting in following steps is performed with weights, which are proportional to differences between the polynomial at specified points computed in the previous iteration and STEC values for individual points. Subsequently, the final polynomial is subtracted in order to eliminate the low-frequency component and to set the background STEC values to 0 . The proposed algorithm allows the simple identification of MSTIDs signatures and minimizes the modification of the STEC time series. Figure 1 presents the example of STEC fluctuations detected for satellite GPS PRN 19 at station DOMA using the proposed algorithm and high-pass filtering executed with Butterworth filter of order 8. In the second case, the cut-off period was set to $2 \mathrm{~h}$. The very good coincidence for both approaches is clearly visible. The shift between the two time series is a consequence of the applied detrending method.

It should be noted that MSTID signatures in the retrieved time series are still dependent on elevation angle and correspond to changes in ionospheric conditions observed in slant directions. Considering the complex structure of the disturbed ionosphere, the patterns of MSTIDs can be expected to be distorted for low-elevated observations. Due to this reason, medium scale disturbances are usually monitored with only high-elevated observations.

The main purpose of the analyses presented in next subsections was the detection of individual ionospheric structures (Section 2.1) and evaluation of STEC discrepancies occurring at medium baselines. (Section 2.2). The presented below analysis focuses on dynamic mutations of MSTIDs patterns, which consequently may affect GNSS positioning performance.

\subsection{MSTIDs detection over Poland area}

The starting point of analysis was the identification of MSTIDs, involving both the amplitude of TEC 


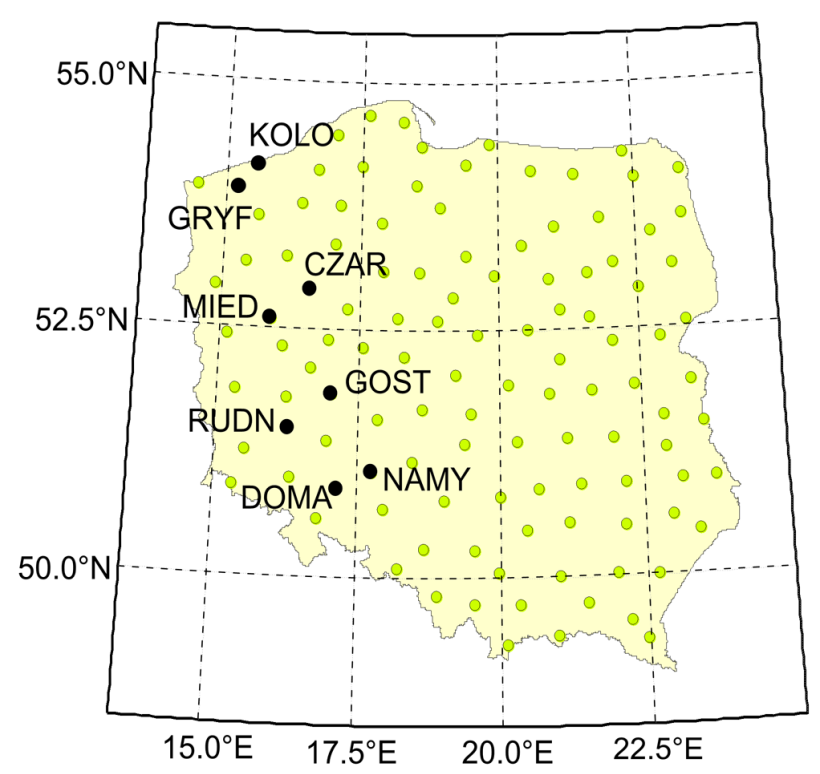

Figure 2. The configuration of TOPNET network with marking of stations used in detailed analyses.

enhancement and the direction of plasma flow. For this purpose GNSS data from the TPI NETpro continuously operating reference network was used (Figure 2). TPI NETpro is the Polish part of the worldwide TopNET Live network and at present consists of about 100 permanent stations tracking GPS+GLONASS observations. The average distance between receivers is 50-60 $\mathrm{km}$, which allows the detection of individual MSTIDs movements all over Poland. In the analysis, the daily measurements from 19.12.2013 were used. The chosen day represents the typical conditions observed day by day in winter months for high solar and low geomagnetic activity respectively. According to the previous research, these months are characterized by intensified MSTIDs in local noon and quiet ionosphere for other hours [Hernández-Pajares et al. 2012]. This fact enables the comparison of different ionospheric conditions and their impact on GNSS positioning. At this place it should be mentioned that during the minimum of solar activity the MSTIDs amplitudes are much lower and the occurrence of these disturbances is less problematic for satellite positioning.

The conducted preliminary study confirmed that the most intense TEC fluctuations appeared in the forenoon hours. In order to depict the structures in the ionosphere, the detrended STEC series were separately computed for each TPI NETpro station according to the above described algorithm. The results for the selected hour (09:30-10:30 UTC) divided into 5-minute periods are presented in Figure 3. It is based on the measurements of two satellites (GPS PRN 18 and GLONASS PRN 19) characterized by the highest elevation angle, exceeding $50^{\circ}$ during the analyzed period. The application of only these satellites narrows the monitored area but more clarifies the view of the individual ionospheric structures. For the purpose of generating maps, STEC values were projected to a single layer model at a height $350 \mathrm{~km}$.

The retrieved results demonstrate the movements of plasma irregularities towards a south-east direction and are converged with previous climatological studies on MSTIDs occurrences [Hernández-Pajares et al. 2012, Otsuka et al. 2013]. In the analyzed period, the maximal amplitude of TEC enhancement slightly exceeded 1 TECU. With regard to individual ionospheric structures, two consecutive MSTID waves can be clearly observed between 09:30 and 10:00 UTC over western and southern Poland moving with a velocity of about $130 \mathrm{~m} / \mathrm{s}$. MSTIDs signatures, depicted as high gradients between STEC values at neighboring stations, are also observed in other parts of the ionosphere, but it is difficult to recognize any specific structures. It is related to relatively small analyzed area, direction of MSTIDs movement and complexity of ionospheric structures. The last of the mentioned factors is probably caused by superposition of different-scale travelling disturbances.

\subsection{The comparison of MSTIDs patterns observed at} medium baselines

According to the analysis presented in the previous subsection, individual MSTIDs are detected more effectively in the ionosphere above south-western Poland. Clearly visible signatures of ionospheric disturbances for this region allow the identification of STEC discrepancies and mutations of MSTIDs patterns observed in different directions. Measurements from eight receivers belonging to the TPI NETpro network were used in order to compare the ionospheric conditions. The stations represent four pairs of receivers: (1) GRYF, KOLO, (2) MIED, CZAR, (3) RUDN, GOST and (4) DOMA, NAMY. Their locations and corresponding baseline lengths are presented in Figure 2 and Table 1 respectively. The baselines of each pair were oriented perpendicular to MSTIDs movements to compare the plasma wave patterns in this direction. In order to retrieve information on time changes in MSTIDs signatures, all four sets of receivers were oriented accordingly to the propagation of ionospheric disturbances. The distances between the receivers in this direction equal about $100 \mathrm{~km}$. Analogously to the previously described research, this analysis was also performed using a detrended STEC time series and the same high-elevated observations. The results for all selected pairs of receivers are presented in Figure 4, which shows the changes in ionospheric conditions observed in $3 \mathrm{~h}$ and $2.75 \mathrm{~h}$ periods for GPS PRN 19 (left panel) and GLONASS PRN 18 (right panel) respectively. The applied cut-off angle 


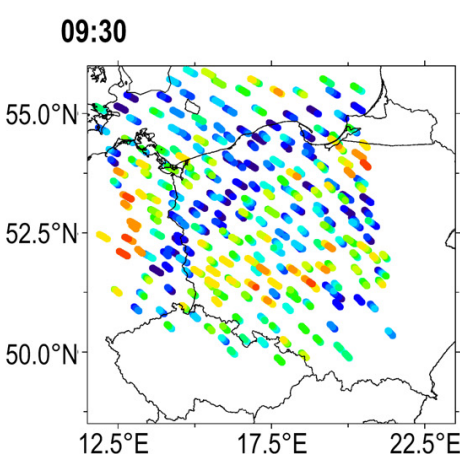

09:45

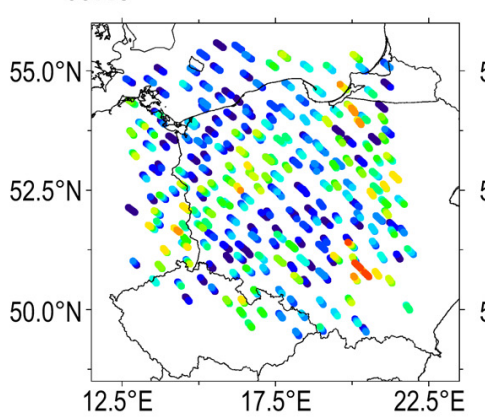

10:00

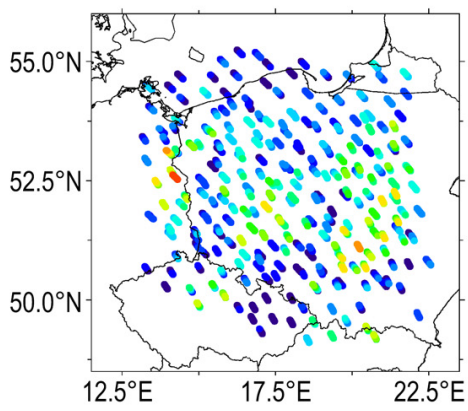

10:15

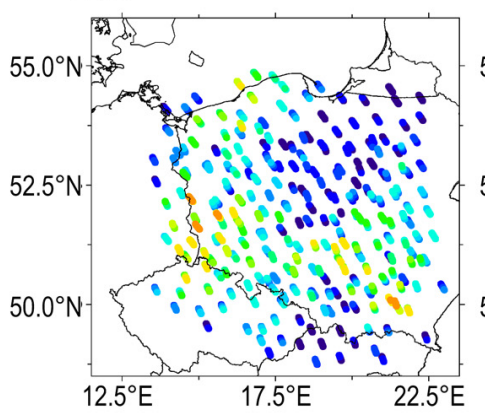

09:35

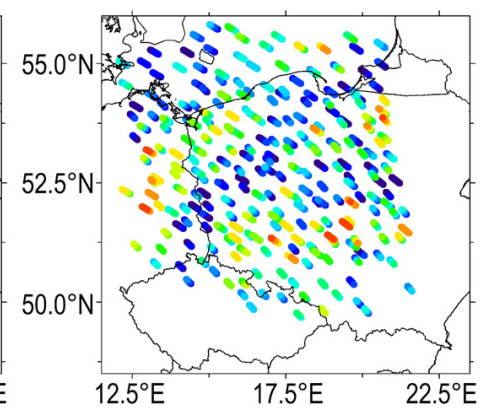

09:50

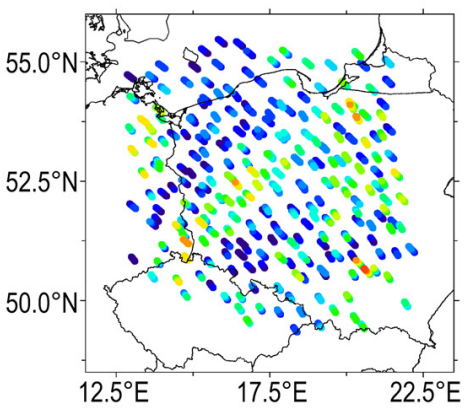

10:05

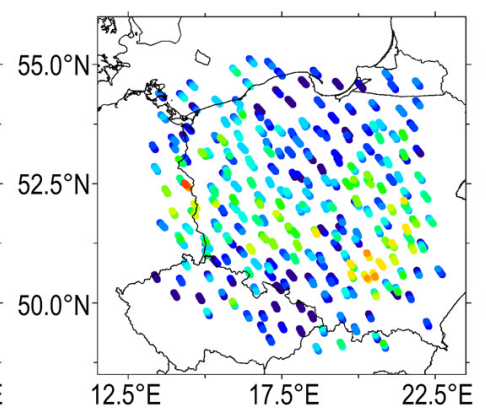

$10: 20$

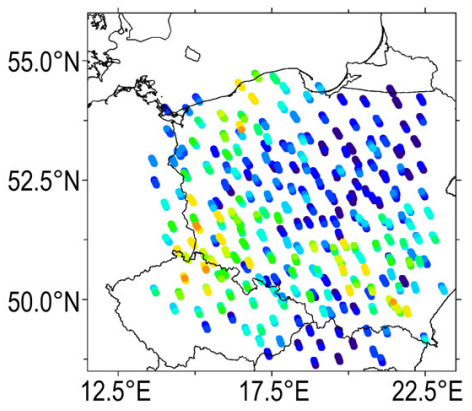

09:40

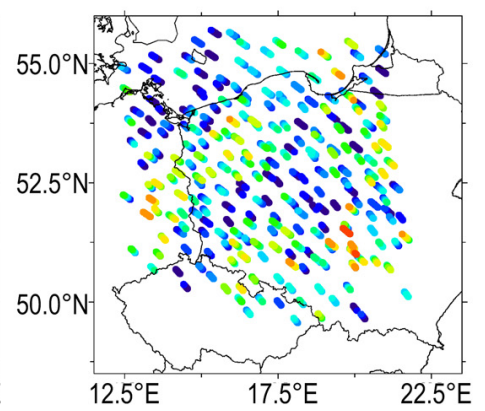

09:55

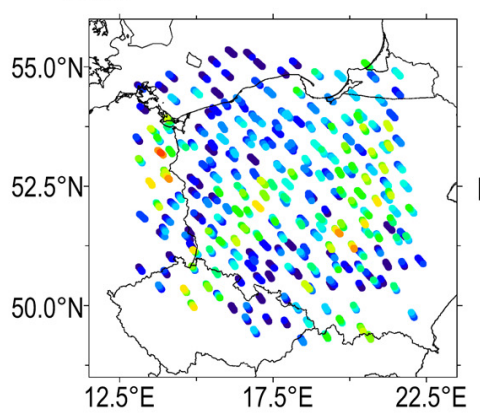

Detrended STEC [TECU]

$>1.4$

- $1.2-1.4$

1.0-1.2

$0.9-1.0$

$0.8-0.9$

$10: 10$

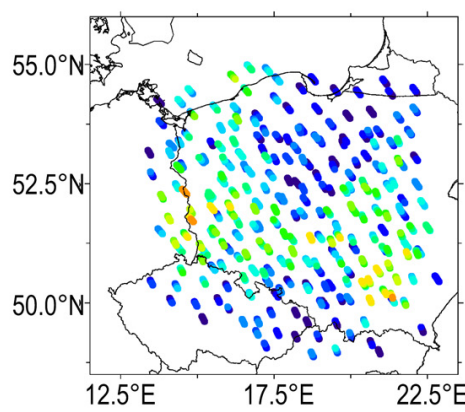

$0.7-0.8$

$0.6-0.7$

$0.5-0.6$

$0.4-0.5$

$0.3-0.4$

$0.2-0.3$

0.1-0.2

- 0.0-0.1

Figure 3. The ionospheric disturbances over Poland area observed with GNSS measurements (GPS PRN 18 and Glonass PRN 19) for period 9:30-10:30 UTC, 19.12.2013.

Stations

Distance $[\mathrm{km}]$

GRYF-KOLO

35.5

MIED-CZAR

56.0

RUDN-GOST

65.1

DOMA-NAMY

47.3

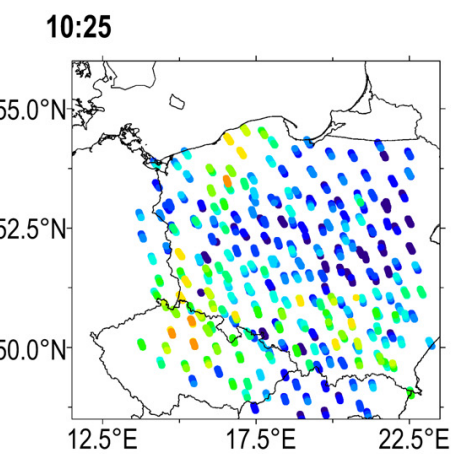



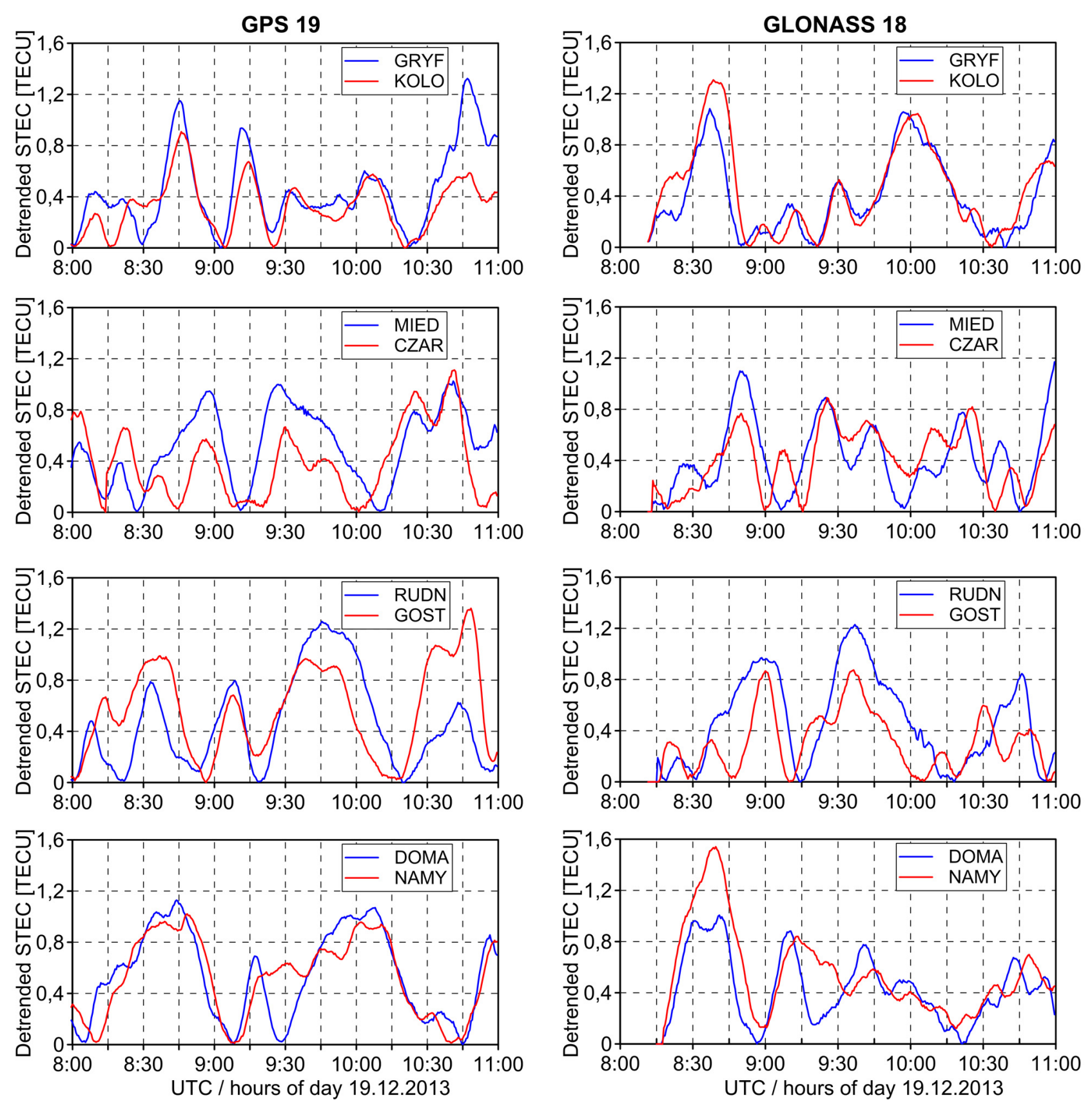

Figure 4. The comparison of STEC fluctuations observed at medium baselines for selected satellites: GPS PRN 19 (left panel) and Glonass PRN 18 (right panel), 08:00-11:00 UTC, 19.12.2013.

ing these results it should be noted that the distance between GRYF and KOLO stations equals only $35.5 \mathrm{~km}$. In the next two cases, MIED-CZAR and RUDN-GOST, the distance is about $60 \mathrm{~km}$, which can be considered as a typical baseline length in permanent networks of GNSS receivers. In these cases, the differences in detected MSTIDs patterns are clearly visible. Attention should be given to signatures of plasma waves which occur only at one of the coupled stations. This effect can be noticed at: 08.45 UTC (GPS PRN 19), 09:10 UTC (GLONASS PRN 18) for the first pair MIED-CZAR and 08:45 UTC (GLONASS PRN 18) in the case of the second. Such differences in STEC time series are expected to be highly problematic even for the interpolation of ionospheric delays based on a reference station solution. Generally the differences between the results often reach 0.4 TECU even at high elevations. The maximal discrepancy between STEC values (0.7 TECU) is observed at 10:30 UTC (GPS PRN 19) for the RUDN-GOST baseline. The last analyzed time series present a comparison of ionospheric conditions over southern Poland (DOMA-NAMY). Similar to the first pair, these results present a good consistency between STEC fluctuations observed at both stations. The high differences in detected values can be noticed only for two periods: 08:30-08:45 UTC (GLONASS PRN 18) and 09:20-09:30 UTC (GPS PRN 19 and GLONASS PRN 18) and they reach up to 0.5-0.6 TECU. Moreover, the increased 
STEC values in the second case are detected simultaneously for both satellites only at the NAMY station.

The comparison of the retrieved STEC time series for all pairs of receivers provides also the information on temporal changes in MSTIDs patterns. Considering that the colocation of different sets of receivers was oriented compatibly with the detected MSTIDs propagation, changes in STEC time series between particular pairs of receivers correspond to time modifications of MSTIDs signatures. The results obtained for both satellites confirm the high variability of these structures in this domain as well, and it is predominantly visible in STEC values retrieved between 09:00-10:00 UTC at the first pair (GRYF-KOLO) and subsequently in measurements shifted in time for the other sets of receivers. In both cases, one can observe the significant modification in plasma wave amplitudes and periods.

In order to provide a complete view of ionospheric conditions, Figure 5 presents the impact of upper parts of the atmosphere during a quiet period. It demonstrates the STEC time series retrieved for satellite GPS PRN 9 between 17:30 and 20:30 UTC. Similar to the presented above analyses, the satellite was observed at high elevation angle (about $50^{\circ}$ at the beginning and at the end of session and almost vertical at 19:00 UTC). The results for 8 selected stations demonstrate that the typical difference for quiet conditions does not exceed 0.1 TECU. The increased discrepancies in the presented sample can be observed only for the shortest baseline (GRYF-KOLO). The differences between STEC time series at 17:30-18:00 UTC and 19:30:19:45 UTC reach up to $0.25-0.3 \mathrm{TECU}$.

A comparison of results obtained for quiet and disturbed period clearly depicts the amplification of discrepancies between STEC values related to MSTIDs occurrences. The results demonstrate that these disturbances often lead to differences amounting to 0.5 TECU at neighboring stations. Thus, in such conditions the double differenced ionospheric delays are expected to exceed the boundary value of STEC discrepancy 0.25 TECU [Hernández-Pajares et al. 2000]. More importantly these differences were observed at high elevations and can be treated as the best-case scenario in comparison to effect observed for low-elevated measurements.

\section{Rapid static positioning in disturbed ionospheric conditions}

The results presented above clearly depict mid-latitudes disturbances as a source of STEC discrepancies often exceeding acceptable level in context of precise positioning. This section is aimed at MSTIDs impact on double differenced ionospheric delays and conse-

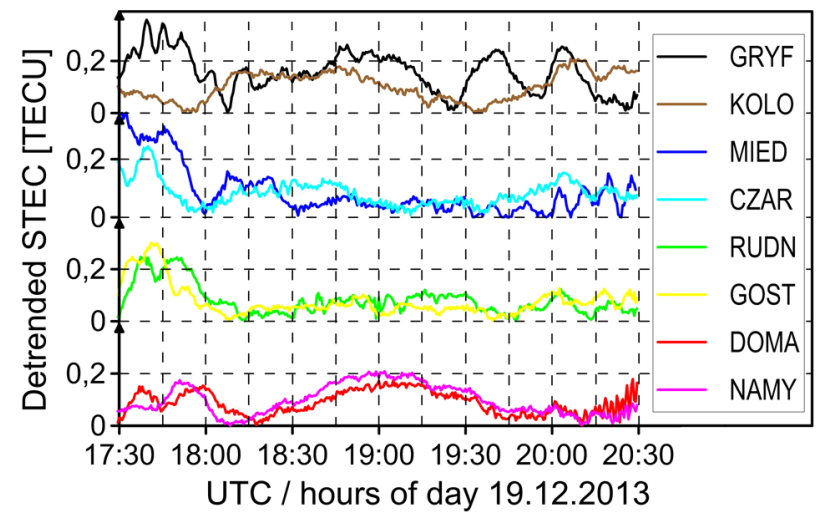

Figure 5. STEC fluctuations during quiet ionospheric conditions observed at eight analyzed stations for satellite GPS PRN 9, 17:3020:30 UTC, 19.12.2013.

quently on GPS rapid static positioning performance. The analysis includes the statistics of coordinate estimation using two strategies. First of them is a classical approach of geometry-based relative positioning with weighted ionosphere and troposphere parameters. The second represents a modification of the former. In the new approach the raw phase observations are corrected using Rate of TEC (ROT) values which allows the elimination of temporal changes of ionospheric delays.

\subsection{Applied algorithm of GNSS positioning}

As a reference precise positioning approach was used the geometry-based relative model with weighted ionosphere and troposphere. It represents one of most commonly used functional models used in navigation and surveying applications. Apart from coordinates, the applied model involves modelling of additional parameters such as: zenith tropospheric delays, double differenced ionospheric delays and double differenced integer ambiguities [Paziewski and Wielgosz 2014]. The estimation process in the applied software algorithm is performed sequentially with linearized observations and pseudo observations [Paziewski 2015a]. The first part of model contains double differenced $(\nabla \Delta)$ carrier phase and pseudorange observations (Equation 2).

$$
\begin{gathered}
\nabla \Delta \lambda_{1} \varphi_{1}=\nabla \Delta \rho+\nabla \Delta T-\nabla \Delta I+\nabla \Delta \lambda_{1} N_{1} \\
\nabla \Delta P_{1}=\nabla \Delta \rho+\nabla \Delta T+\nabla \Delta I \\
\nabla \Delta \lambda_{2} \varphi_{2}=\nabla \Delta \rho+\nabla \Delta T-\nabla \Delta \frac{f_{1}^{2}}{f_{2}^{2}} I+\nabla \Delta \lambda_{2} N_{2} \\
\nabla \Delta P_{2}=\Delta \rho+\nabla \Delta T+\nabla \Delta \frac{f_{1}^{2}}{f_{2}^{2}} I
\end{gathered}
$$

$\lambda_{1}, \lambda_{2}$ - signals wavelengths

$f_{1}, f_{2}$ - signals frequencies

$\varphi_{1}, \varphi_{2}, P_{1}, P_{2}$ - dual-frequency carrier phase and code measurements respectively

$\rho$ - geometric distance

$T$ - tropospheric delay

$I$ - ionospheric delay referred to first frequency

$N_{1}, N_{2}$ - integer ambiguities 
$\nabla \Delta T=\left(\alpha^{m}{ }_{k} Z T D_{k}-\alpha_{k}^{n} Z_{k} D_{k}-\alpha^{m}{ }_{l} Z T D_{l}+\alpha^{n}{ }_{l} \mathrm{ZTD}_{l}\right)$ $Z T D_{k}, Z T D_{l}$ - zenith tropospheric delays estimated separately for stations $k$ and $l$

$\alpha$ - troposphere mapping function coefficient (superscripts $m$ and $n$ denote satellites).

The second part of the model represents ionospheric and tropospheric pseudo observations (Equation 3), which allow constraining selected parameters using a priori variance factors.

$$
\begin{aligned}
\nabla \Delta I & =\nabla \Delta I^{\prime} \\
Z T^{\prime} D_{k} & =Z T D_{k}^{\prime}
\end{aligned}
$$

These equations contain the estimated parameters $\left(\nabla \Delta I\right.$ and $\left.Z T D_{k}\right)$ and their a priori values $\left(\nabla \Delta I^{\prime}\right.$ and $\left.Z T D_{k}^{\prime}\right)$ respectively. In presented analyses it was assumed that a priori values of double differenced ionospheric delays equal 0 . In this assumption the model is commonly termed as ionosphere-float. The a priori values of tropospheric delays were adopted from UNB3m empirical model [Leandro et al. 2008].

As a result the applied observational model is resolved applying least squares adjustment with $a$ priori parameters constraining [Xu 2007]:

$$
\left(A^{T} P_{L} A+B^{T} P_{w} B\right) d x-\left(A^{T} P_{L} L+B^{T} P_{W} W\right)=0
$$

where:

$A, B$ - design matrices for observations and pseudo observations

$L, W$ - observed minus computed vectors for observations and pseudo observations

$P_{L}, P_{W}$ - observations and pseudo observations weight matrices

$d x$ - estimated corrections to the $a$ priori values of parameters.

In the model presented above the weight matrix composition is realized using zenith-referenced standard deviations of undifferenced code and phase observations at the level of $0.3 \mathrm{~m}$ and $0.003 \mathrm{~m}$ respectively. The elevation-dependent weighting of observations is performed using function:

$$
w(e)=\sin ^{2}(e)
$$

The corresponding values of standard deviations for the tropospheric and ionospheric pseudo observations are set to $0.003 \mathrm{~m}$ and $0.05 \mathrm{~m}$. These values were set on the basis of earlier empirical studies [Wielgosz et al. 2005].

The process of parameters estimation was executed in the three steps procedure: float solution with real values of ambiguities; integer ambiguity resolution with
MLAMBDA method [Chang et al. 2005]; and finally, the so called fixed solution with eliminated known values of integer ambiguities. The rover coordinate estimation was executed in this study in multi-baseline mode, i.e. in common processing of all vectors. In order to compute satellite positions the broadcast ephemerides were used.

\subsection{Rate of TEC corrections}

In the standard weighted model a new slant ionospheric delay parameter is introduced at each epoch for each double differenced observation. This causes rapid growth of the number of parameters correspondingly to the session length. The proposed strategy eliminates temporal fluctuations of ionospheric delays what implies the reduction of ionospheric parameters to one for each arc of double differenced observations. Since its comprehensive description can be found in Sieradzki and Paziewski [2015], only a brief one is introduced below.

The starting point for this recently proposed algorithm is a difference of geometry free linear combinations between two epochs $i$ and $j$, often called Rate of TEC - ROT (Equation 6).

$$
\begin{aligned}
R O T_{t j i}= & L_{4_{t j}}-L_{4_{t i}}=I_{4_{t j}}+\lambda_{1} N_{1}-\lambda_{2} N_{2}- \\
& -\left(I_{t_{t i}}+\lambda_{1} N_{1}-\lambda_{2} N_{2}\right)=\Delta I_{t_{t j i}}
\end{aligned}
$$

Providing the constant ambiguity terms, the ROT values represent directly the temporal change of ionospheric delay between any two epochs related to the geometry free combination $\left(\Delta I_{t_{t j i}}\right)$. The application of appropriate conversions allows the retrieval of ionospheric delay changes corresponding to base signals $\mathrm{L}_{1}$ and $\mathrm{L}_{2}$ (Equations 7 and 8).

$$
\begin{aligned}
\Delta I_{1_{t j i}} & =\frac{f_{2}^{2}}{f_{2}^{2}-f_{1}^{2}} * \Delta I_{t_{t j j i}} \\
\Delta I_{t_{\mathrm{tji}}} & =\frac{f_{1}^{2}}{f_{2}^{2}-f_{1}^{2}} * \Delta I_{t_{t j i}}
\end{aligned}
$$

Finally, the addition of ionospheric delay changes $\left(\Delta I_{1_{t j i}}\right)$ and $\left(\Delta I_{2_{t j j}}\right)$ to raw $\mathrm{L}_{1}$ and $\mathrm{L}_{2}$ observations at epoch i results in identical ionospheric delays at both epochs $i$ and $j$.

$$
\begin{aligned}
& \widetilde{L}_{1_{t i}}=L_{1_{t i}}-\Delta I_{1_{t j i}} \\
& \widetilde{L}_{2_{t i}}=L_{2_{t i}}-\Delta I_{2_{t j i}}
\end{aligned}
$$

After this step, the corrected observations at epoch $i\left(\widetilde{L}_{1_{t i}}, \widetilde{L}_{t_{t i}}\right)$ are affected by the same ionospheric delay as at epoch $j$. This process can be executed for any two epochs in the session, assuming lack of cycle-slips. The application of these corrections to all undifferenced observations of the specified satellite results in identical ionospheric delays for the entire arc. Consequently, also 


\begin{tabular}{cccccc} 
Station & $\mathbf{B}\left[^{\circ}\right]$ & $\mathbf{L}\left[^{\circ}\right]$ & $\mathbf{H}[\mathbf{m}]$ & Receiver & Antenna \\
\hline DOMA & $\mathrm{N} 50.89267$ & E 17.13358 & 195.33 & Topcon NET-G3A & CR-G5 \\
GOCZ & $\mathrm{N} 51.00283$ & E 16.32524 & 253.95 & Topcon NET-G3A & CR-G5 \\
KLDZ & $\mathrm{N} 50.43557$ & E 16.65189 & 359.5 & Trimble NetR9 & TRM59900.00 \\
OPOL & $\mathrm{N} 50.69498$ & E 17.95378 & 211.00 & Topcon NET-G3A & CR-G5 \\
PRUS & $\mathrm{N} 51.37535$ & E 16.95474 & 163.79 & Topcon NET-G3A & CR-G5 \\
\hline
\end{tabular}

Table 2. Stations details.

the double-differenced ionospheric delays are constant and can be estimated not as epoch dependent but as constant parameters per session for particular arc [Sieradzki and Paziewski 2015]. It should be remarked that algorithm does not eliminate ionospheric delays but only their temporal fluctuations. The efficiency of algorithm is related to magnitude of double differenced ionospheric delays observed at the reference epoch(s). The reference epoch can be different for each satellite which allows the more effective reduction of ionospheric delays and improvement of ambiguity fixing. Based on the preliminary empirical studies, the epochs characterized by minimal L4 values of undifferenced observations were chosen as references in this work.

\subsection{Experiment design}

In order to evaluate the impact of MSTIDs on rapid static GPS positioning, the test network located in the southern Poland was chosen. It consist of five permanent stations. Four of them (DOMA, GOCZ, PRUS, OPOL) belong to the TPI NETpro network and the last (KLDZ) is a part of the Polish national active reference network - ASG-EUPOS [Bosy et al. 2007]. In this experiment, the DOMA station, in the middle of test network, serves as a rover receiver, and the other receivers are

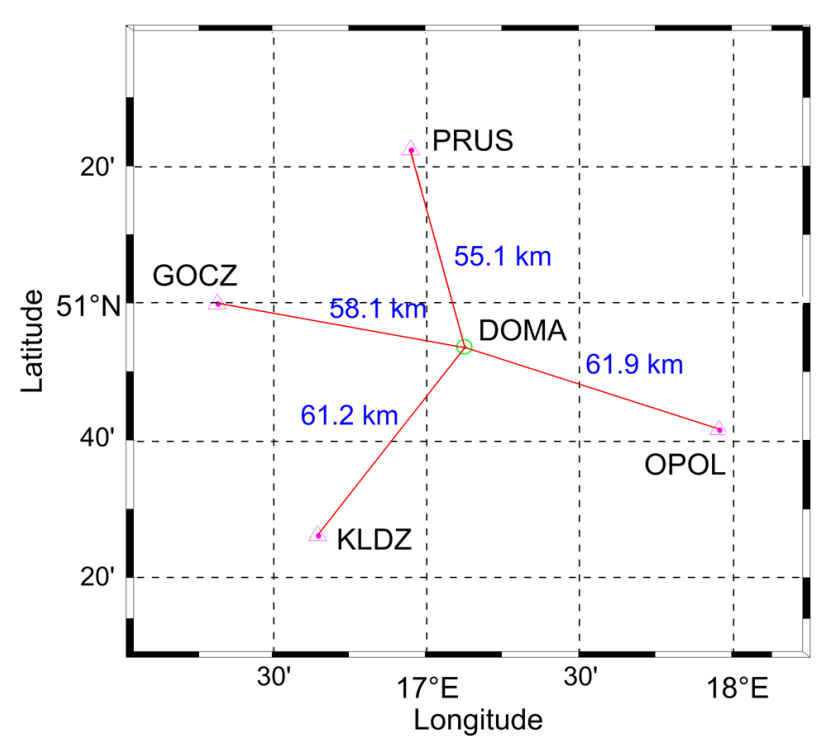

Figure 6. Test network. treated as reference sites. The average baseline length between the rover and reference points is about $58 \mathrm{~km}$. More detailed information on locations and equipment of particular stations can be found in Table 2 and Figure 6.

In order to confirm the repeatability of ionospheric conditions and efficiency of the proposed algorithm, the analysis of positioning covers three days: the analyzed in the previous section 19.12.2013 and two consecutive 20-21.12.2013. According to the tests presented above, the amplified TEC fluctuations were observed only in selected hours. Thus, the daily study for each day enables the comparison of results retrieved in quiet and disturbed periods. The analysis presented in the next sections focuses firstly on the impact of MSTIDs on magnitude of double-differenced ionospheric delays observed at different elevation angles and secondly on a comparison of the performance of rapid static GPS positioning for standard and modified algorithms. In both cases there were used measurements with $30 \mathrm{~s}$ interval. The positioning was executed for epochs with number of satellites higher than 5 .

\subsection{Impact of TEC fluctuations on double differenced} ionospheric delays

Prior to the main analyses of positioning performance in disturbed ionospheric conditions, it is advisable to present their impact on double differenced GPS observations. For this purpose, the integer values of double-differenced ambiguities were resolved in two hour sessions for each baseline of the analyzed network (Figure 6). Their true values allowed the retrieval of information on real ionospheric delays using a geometryfree combination. Thus, the changes of conditions in the upper atmosphere can be followed epoch by epoch for each double differenced arc. Such a distribution of ionospheric delays as a time function is presented in Figure 7 for all analyzed days. The ionospheric parameters retrieved for particular baselines are expressed in meters, which is more appropriate and widely used with regards to satellite positioning ( 1 TECU corresponds to delays about $0.16 \mathrm{~m}$ and $0.26 \mathrm{~m}$ on $\mathrm{L} 1$ and $\mathrm{L} 2$ respectively). The small gaps in the presented time series of 

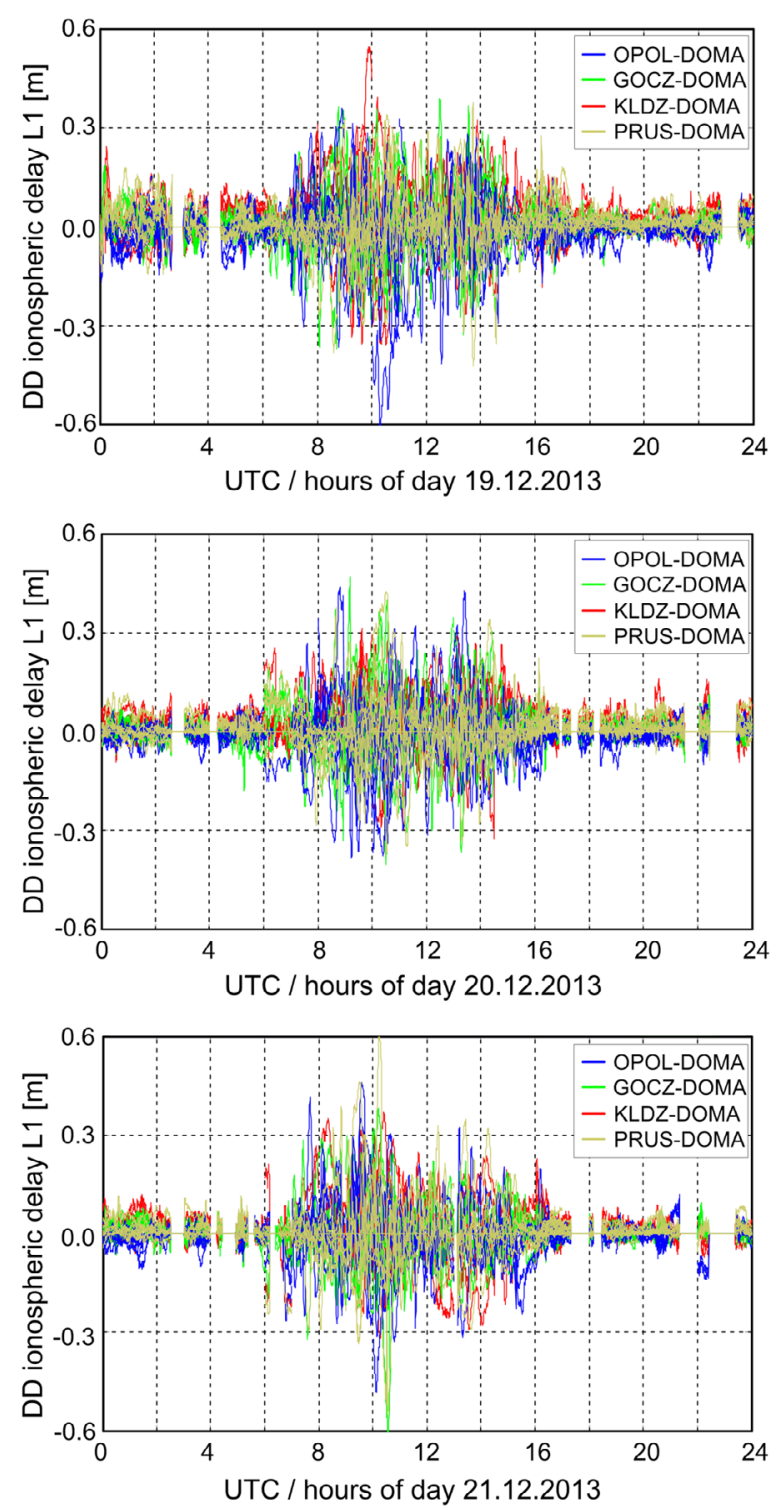

Figure 7 (top to bottom). Double differenced ionospheric delays on L1 frequency for all baselines obtained from geometry-free solution with fixed ambiguities (19.12.2013 - top panel, 20.12.2013 - middle panel, 21.12.2013 - bottom panel).

ionospheric delays are caused by too low number of satellites or lack of observations for selected epochs. The second reason appears for days 20-21.12.2013 and is an effect of interruption in data stream used for RINEX files generation. The results for three consecutive days clearly depict the increase of double-differenced ionospheric delays occurring at selected hours between 07:00 and 15:00 UTC. Furthermore, they confirm the daily pattern of MSTIDs occurrence and related with it repeatability of ionospheric conditions. The highest values of the double-differenced ionospheric delays are observed at about 10:00 UTC. Their amplitudes exceed in extreme cases $0.5 \mathrm{~m}$, which corresponds to about 2.5 cycle of L1 measurements. Presumably the observed effect may significantly affect the ambiguity resolution during disturbed periods. In the
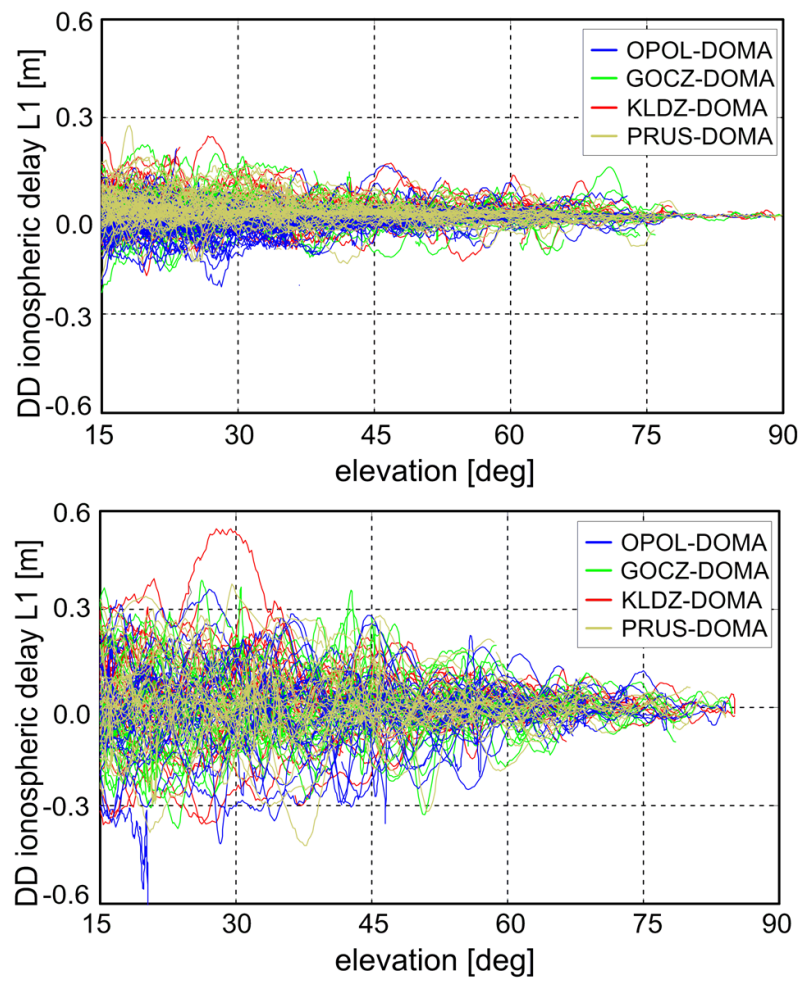

Figure 8 (top to bottom). The dependence of double differenced ionospheric delays on elevation angle during quiet (top panel) and disturbed conditions (bottom panel), 19.12.2013.

case of quiet periods (00:00-07:00UTC and 15:00-24:00 UTC, 19-21.12.2013), the values of double-differenced ionospheric delays in a specific epochs do not exceed several centimeters.

The results presented in Figure 7 depict also the enhanced ionospheric impact in comparison to the effect observed at high elevation observations in Section 2. This growth detected in double-differenced ionospheric delays is primarily related to low-elevated observations used for satellite positioning. The discrepancies observed in such cases are amplified by the complex structure of the ionosphere disturbed by MSTIDs occurrences. An example of double differenced ionospheric delay distributions as a function of elevation angle are presented in Figure 8 separately for quiet (00:00-07:00 UTC and 15:00-24:00 UTC, 19.12.2013 - top panel) and disturbed hours (07:00-15:00 UTC, 19.12.2013 - bottom panel). A value corresponding to the second satellite, apart from the reference one, was taken as an indicator of elevation angle. The reference satellite's elevation is fundamentally the highest possible and its impact is assumed to be meaningless. The results for quiet periods clearly demonstrate that the double differenced ionospheric delays do not usually exceed several centimeters in the analyzed case. Moreover, the growth of these values at low-elevated observations is relatively weak. Analysis of the disturbed conditions shows a significant growth of real ionospheric delays, which starts at $45-50^{\circ}$ of el- 
evation angle. In the case of angles below $45^{\circ}$ the detected values often reached $0.25 \mathrm{~m}$. The presented results of double-differenced ionospheric delays explicitly depict the scale of deteriorating impact of MSTIDs, featured only by 1 TECU peak-to-peak values observed in a nearly vertical direction.

\subsection{Rapid static positioning performance}

In order to examine the performance of rapid GPS positioning in different ionospheric conditions, the daily data from all three days (19-21.12.2013) was divided into 5 minute periods. Taking into account a 30 -second interval of data, particular sessions consisted of ten measurement epochs. Each of them was processed twice using both introduced strategies: standard ionosphereweighted model (IW) and Rate of TEC corrections algorithm (RTC). As it was mentioned the processing of GNSS data was executed only when the number of satellites was six or higher, so a few short periods in the quiet hours were not considered in statistics. The posi- tioning performance was evaluated using a few parameters describing its precision and reliability. Regarding coordinate domain, each component was characterized by standard deviation and mean residual between individual session solutions and reference values. These calculations involved only sessions with correctly resolved ambiguities. In case of ambiguity resolution domain, the ratio of sessions with correctly resolved ambiguities to the total number of sessions (ambiguity success rate - ASR) as well as number of epochs required for obtaining correct solution (time to first fix - TTF) were used as indicators.

Figure 9 presents an example of coordinate residuals obtained in multi-baseline mode for the standard model and its modification using ROT corrections (left and right panel respectively). The positioning performance indicators corresponding to particular analyses were placed in the right top corners. The top panel compares the estimation of horizontal components. The retrieved results show similar and very low aver-
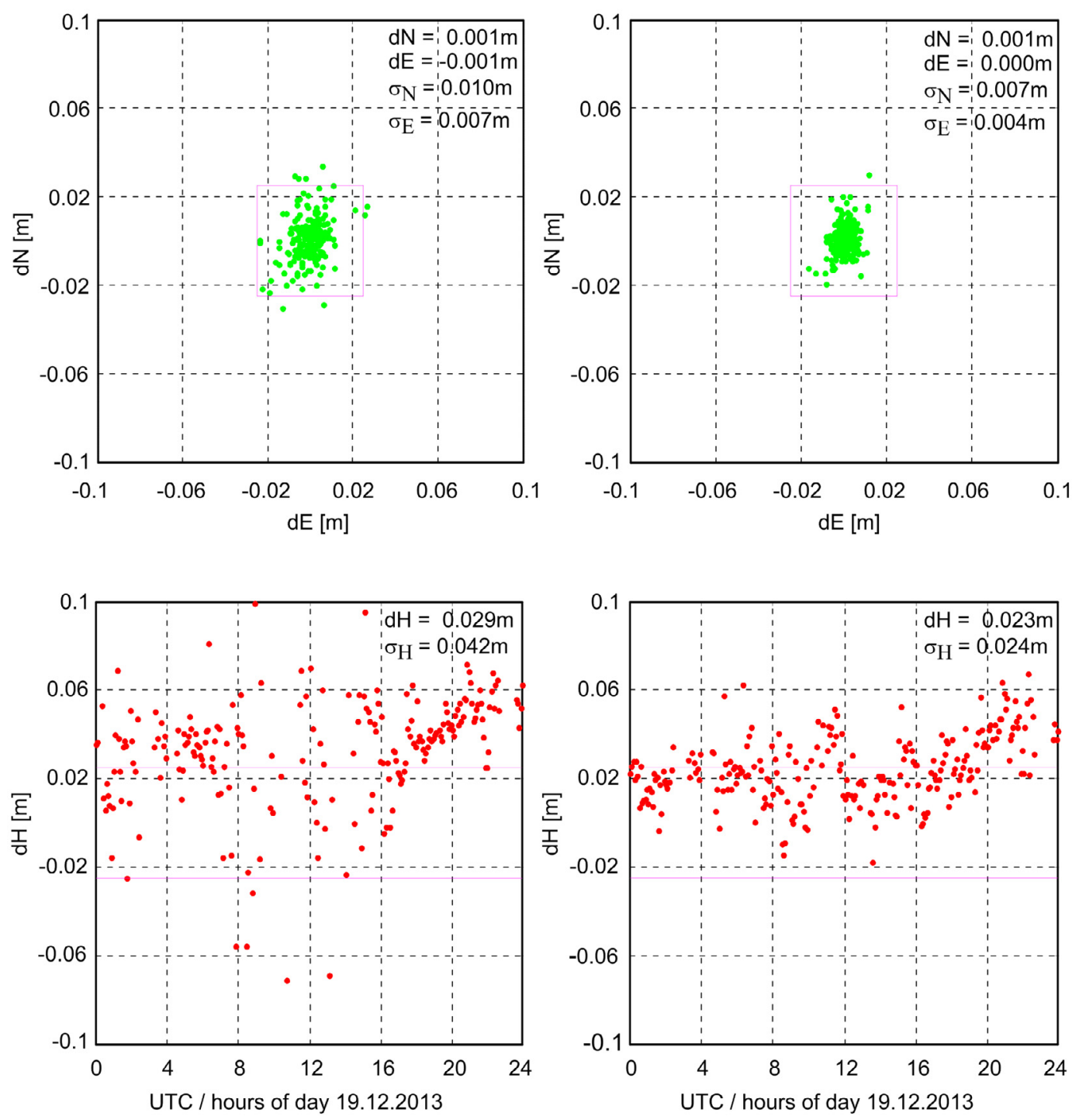

Figure 9. Coordinate residuals for IW model and RTC algorithm, 19.12.2013 (respectively left and right panel). 
age residuals, not exceeding $\pm 0.1 \mathrm{~cm}$, of both strategies in respect to reference coordinates. Concerning the precision described using standard deviations, it can be clearly seen that the modified algorithm improved the results for both horizontal components; the precision for the east one equals $0.7 \mathrm{~cm}$ and $0.4 \mathrm{~cm}$ for standard and modified algorithm respectively. The improved positioning quality is also visible in the spatial distributions presented in the corresponding figures. The results of height component as a function of time clarify the effect of MSTIDs occurrence on satellite positioning (Figure 9, bottom panel). First of all, the repeatability and reliability of the standard approach are much worse in disturbed hours. The increased ionospheric delays reported in previous section caused the high residual values (up to $10 \mathrm{~cm}$ ) for some sessions. The number of correctly resolved sessions clearly drop during the hours affected by MSTIDs. With regard to the new algorithm, the impact of ionospheric disturbances is significantly reduced, and as a result one can observe the improvement of both analyzed indicators. Particularly remarkable are the results for obtained precision. The standard deviations of the height component equal $4.2 \mathrm{~cm}$ and 2.4 $\mathrm{cm}$ for standard and modified algorithm, respectively.

The summary of positioning performance in coordinate domain for three analyzed days is given in Table 3. The results obtained for both algorithms confirm the high accuracy of horizontal components. However, the application of proposed modification improves their precision and leads to reduction of particular stan- dard deviations by $0.3 \mathrm{~cm}$. Even more significant effects can be observed for estimated height, where ROT corrections enhance the precision nearly in half and reduce the mean residual effect by $0.5 \mathrm{~cm}$ as well. Nevertheless, the systematic shift of vertical component is clearly visible for both algorithms. The possible explanation of this fact is the application of widely-used but also relatively simple elevation-dependent weighting function, which can deteriorate the height estimation [Jin et al. 2005]. More detailed analysis of this effect needs further investigations.

Next analyzed indicator is the ambiguity success rate which describes the reliability of ambiguity resolution process in precise GPS positioning. As a resolved sessions in the multi-baseline model are treated these with correct ambiguities for all vectors. In order to highlight the differences in the efficiency of both algorithms, the analyzed days were divided into three periods: 00:00-07:00 UTC, 07:00-15:00 UTC and 15:00-24:00 UTC. Two of them (the first and the last) correspond to quiet ionosphere; the middle one (15:00-24:00 UTC) was affected by MSTIDs occurrence and can be treated as a representative example of positioning in the disturbed conditions. The detailed results of ASR parameter for specified periods during three analyzed days are presented in Table 4.

Considering the quiet conditions, the performance of standard and modified algorithms are at the same, high level. Excluding the sessions with five or less satellites, ASR exceeds $97 \%$ in quiet periods for all analyzed

\begin{tabular}{|c|c|c|c|c|c|c|c|}
\hline \multirow{2}{*}{ Day } & \multirow{2}{*}{ Strategy } & \multicolumn{2}{|c|}{$\mathrm{N}[\mathrm{cm}]$} & \multicolumn{2}{|c|}{$\mathrm{E}[\mathrm{cm}]$} & \multicolumn{2}{|c|}{$\mathrm{U}[\mathrm{cm}]$} \\
\hline & & $\mathrm{dN}$ & $\mathrm{N}$ & $\mathrm{dE}$ & $E$ & $d U$ & $\mathrm{U}$ \\
\hline \multirow{2}{*}{19.12 .2013} & IW & 0.1 & 1.0 & -0.1 & 0.7 & 2.9 & 4.2 \\
\hline & RTC & 0.1 & 0.7 & 0.0 & 0.4 & 2.3 & 2.4 \\
\hline \multirow{2}{*}{20.12 .2013} & IW & 0.0 & 0.9 & 0.0 & 0.7 & 3.2 & 4.5 \\
\hline & RTC & 0.0 & 0.7 & 0.1 & 0.4 & 2.8 & 3.0 \\
\hline \multirow{2}{*}{21.12 .2013} & IW & 0.0 & 0.9 & 0.0 & 0.6 & 2.9 & 3.0 \\
\hline & RTC & 0.0 & 0.7 & -0.1 & 0.4 & 2.4 & 1.1 \\
\hline
\end{tabular}

Table 3. Statistics of rapid static positioning.

\begin{tabular}{ccccc}
\multirow{2}{*}{ Day } & \multirow{2}{*}{ Strategy } & \multicolumn{3}{c}{ ASR [\%] } \\
\cline { 3 - 5 } 19.12 .2013 & IW & $00-07$ UTC & 07-15 UTC & $15-24$ UTC \\
\hline \multirow{2}{*}{20.12 .2013} & RTC & 97 & 56 & 99 \\
& IW & 99 & 68 & 100 \\
\hline \multirow{2}{*}{21.12 .2013} & RTC & 99 & 89 & 100 \\
& IW & 100 & 57 & 99 \\
\hline
\end{tabular}

Table 4. Ambiguity success rate at selected periods. 
days. Based on these results, both algorithms are concluded to have similarly efficiency of ambiguity fixing during such conditions. The results obtained for the disturbed periods clearly depict the discrepancy in ambiguity fixing between both applied strategies. The ASR ratio with the standard ionosphere-weighted model varies from $56 \%$ to $65 \%$, which confirms the low reliability of this approach in periods affected by MSTIDs occurrence. In contrast to this, one can observe the significant improvement of ambiguity success rate for ROT correction algorithm up to $90 \%$. Furthermore, this progress in ambiguity fixing is observed for each day, what basically proves the better efficiency of the proposed modification.

In order to highlight the impact of ionospheric disturbances on ambiguity fixing Figure 10 presents the time-to-fix values obtained in specified 5 -minute sessions for both analyzed strategies. It is clearly visible that for the quiet conditions in most of sessions ambiguities were correctly resolved in the first epoch, what generally confirms the high efficiency of ionosphereweighted model supported by MLAMBDA method. The occurrence of medium scale ionospheric disturbances at noon hours significantly extended the fixing time for both strategy. However, one can observe a larger number of session resolved for consecutive epochs in the case of the modified algorithm (bottom panels for each day). On the other hand, these sessions were often unresolved in case of the standard positioning approach (top panels).

\section{Summary and conclusions}

The impact of dynamic TEC changes on precise GNSS positioning is challenging and still an open problem. In the case of mid-latitudes, the observed TEC fluctuations are mostly associated with MSTIDs. The presented results confirm that their occurrence leads to a complex structure of the ionosphere which is especially undesired with regard to rapid GNSS positioning. The results clearly demonstrate that MSTIDs signatures observed at medium baselines may greatly differ including both the amplitude of TEC enhancement and shape of wave. Thus, the modelling of disturbed ionosphere is extremely difficult and would require the simultaneous processing of large data set. Further investigations on double-differenced ionospheric delays depict the amplification of MSTIDs impact at lower elevation angles, which can be significant even at $45^{\circ}$ of elevation angle. As a result of this, MSTIDs characterized by peak-to-peak values with magnitude about 1 TECU may cause growth in double-differenced ionospheric delays up to $0.5 \mathrm{~m}$ in extreme cases. Moreover, the analysis covering three consecutive days confirms
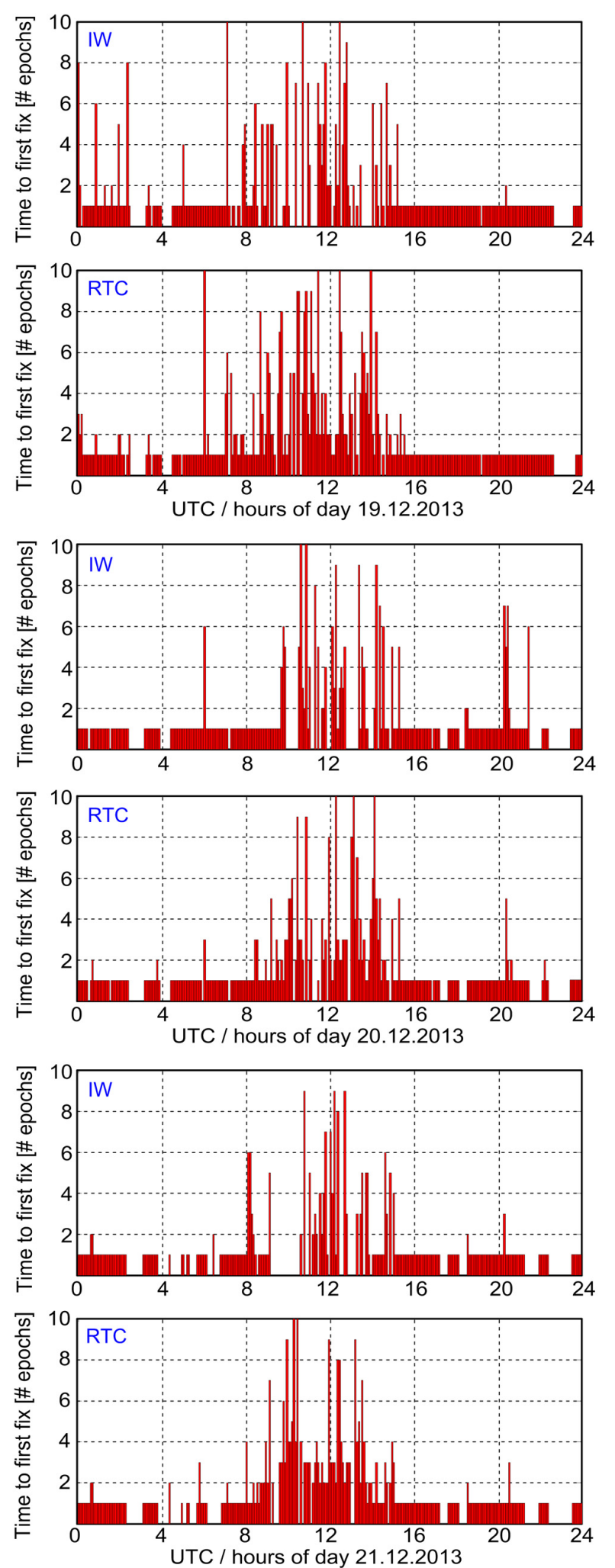

Figure 10. Time to first fix for IW model and RTC algorithm (top and bottom panels for each analysed day respectively).

the daily pattern of MSTIDs occurrence, which strongly affect the reliability of ambiguity fixing for the standard ionosphere weighted model. In the this case, the ASR ratio dropped to about $60 \%$, which clearly demonstrates the scale of the problem, particularly if we consider the frequency of MSTIDs occurrence at mid-latitudes. 
In order to mitigate the impact of ionospheric disturbances, the authors have proposed the modification of the GPS positioning algorithm using ROT corrections. This algorithm allows the reduction of epoch-varying ionospheric delays to one selected reference epoch and the elimination of the temporal TEC fluctuations occurring in phase observations. As a consequence it is possible to estimate the double-differenced ionospheric delays as a constant parameter during the session. The first results show that the application of ROT corrections significantly improves ambiguity fixing during disturbed ionosphere periods. During these periods, the average growth of ASR from about $60 \%$ to almost $90 \%$ can be observed.

This preliminary study presents very promising results of the mitigation of MSTIDs impact on GPS positioning using new approach. The proposed algorithm does not require any an external modelling of ionosphere conditions and can be easily implemented in multi-GNSS positioning. The latter aspect should allow further improvement of coordinate estimation performance going beyond the effects related to larger number of observations [Paziewski and Wielgosz 2014, Li et al. 2015b]. It is worth noting that ROT corrections can be applied to relative and single precise point positioning as well. Future studies on selected aspects concerning the presented algorithm such as: its application in multi-GNSS relative and absolute positioning and the optimization of reference epoch choice should lead to improved GNSS positioning reliability based on the introduced method.

Acknowledgements. The authors are grateful for GNSS data provided by TPI NETpro and ASG-EUPOS services.

\section{References}

Alves da Silva, H., P. Camargo, J.F.G. Monico, M. Aquino, H.A. Marques, G.G. De Franceschi and A. Dodson (2010). Stochastic modelling considering ionospheric scintillation effects on GNSS relative and point positioning, Adv. Space Res., 45 (9), 11131121; http:// dx.doi.org/10.1016/j.asr.2009.10.009.

Aquino, M., J.F.G Monico, A.H. Dodson, H.A. Marques, G.G. De Franceschi, L. Alfonsi, V. Romano and M. Andreotti (2009). Improving the GNSS positioning stochastic model in the presence of ionospheric scintillation, J. Geodesy, 83 (10), 953-966; http:/ / dx.doi.org/10.1007/s00190-009-0313-6.

Bosy, J., W. Graszka and M. Leonczyk (2007). ASGEUPOS - a multifunctional precise satellite positioning system in Poland, European Journal of Navigation, 5 (4), 2-6.

Cellmer, S., J. Paziewski and P. Wielgosz (2013). Fast and precise positioning using MAFA method and new GPS and Galileo signals, Acta Geodyn. Geo- mater., v. 10, No. 4 (172); http:/ / dx.doi.org/10.131 68/ AGG.2013.0038.

Chang, X-W., X. Yang and T. Zhou (2005). MLAMBDA: A modified LAMBDA method for integer leastsquares estimation, J. Geodesy, 79, 552-565; doi:10.1 007/s00190-005-0004-x.

Crocetto, N., F. Pingue, S. Ponte, G. Pugliano and V. Sepe (2008). Ionospheric error analysis in GPS measurements, Annals of Geophysics, 51 (4), 585-595.

Dawidowicz, K., and G. Krzan (2014). Accuracy of single receiver static GNSS measurements under conditions of limited satellite availability, Surv. Rev., 46 (337); http:/ / dx.doi.org/10.1179/1752270613Y.00000 00082.

Garcia-Rigo, A., E. Monte, M. Hernández-Pajares, J.M Juan, J. Sanz, A. Aragon-Angel and D. Salazar (2011). Global prediction of the vertical total electron content of the ionosphere based on GPS data, Radio Sci., 46 (RS0D25); http:/ / dx.doi.org/10.1029/2010 RS004643.

Gioia, C., D. Borio, A. Angrisano, S. Gaglione and J. Fortuny-Guasch (2015a). A Galileo IOV assessment: measurement and position domain, GPS Solut., 19, 197-199; doi:10.1007/s10291-014-0379-3.

Gioia, C., A. Aragon-Angel and J. Fortuny (2015b). GNSS ionospheric model performance under nonnominal conditions: CMEs and Solar Eclipse. 2015 International Association of Institutes of Navigation World Congress Prague, Czech Republic, October 20-23, 2015.

Hernández-Pajares, M., J.M. Juan, J. Sanz and and O.L. Colombo (2000). Application of ionospheric tomography to real-time GPS carrier-phase ambiguities resolution, at scales of $400-1000 \mathrm{~km}$ and with high geomagnetic activity, Geophys. Res. Lett., 27 (13), 2009-2012.

Hernández-Pajares, M., J.M. Juan and J. Sanz (2006a). Real time MSTIDs modeling and application to improve the precise GPS and GALILEO navigation, Proc. the ION GNSS Meeting, Fort Worth, TX, 1358-1368.

Hernández-Pajares, M., J.M. Juan and J. Sanz (2006b). Medium-scale traveling ionospheric disturbances affecting GPS measurements: Spatial and temporal analysis, J. Geophys. Res., 111, A07S11; http:// dx. doi.org/10.1029/2005JA011474.

Hernández-Pajares, M., J.M Juan, J. Sanz and A. Aragon-Angel (2012). Propagation of medium scale travelling ionospheric disturbances at different latitudes and solar cycle conditions, Radio Sci., 47, RS0K05; http:/ / dx.doi.org/10.1029/2011RS004951.

Hu, G., D.A. Abbey, W.E. Castleden, C. Earls, O. Ovstedal and D. Weihing (2005). An approach for 
instantaneous ambiguity resolution for medium- to long-range multiple reference station, GPS Solut., 9, 1-11; http: / / dx.doi.org/10.1007/s10291-004-0120-8.

Jacobsen, K.S., and S. Schäfer (2012). Observed effects of a geomagnetic storm on an RTK positioning network at high latitudes, J. Space Weather Space Clim., 2, A13; http: / / dx.doi.org/10.1051/swsc/2012013.

Jakowski, N., C. Borries and V. Wilken (2012a). Introducing a Disturbance Ionosphere Index (DIX), Radio Sci., 47; http: / / dx.doi.org/10.1029/2011RS004939.

Jakowski, N., Y. Béniguel, G. De Franceschi, M. Hernández-Pajares, K.S. Jacobsen, I. Stanislawska, L. Tomasik, R. Warnant and G. Wautelet (2012b). Monitoring, tracking and forecasting ionospheric perturbations using GNSS techniques, J. Space Weather Space Clim., 2 (A22); http: / dx.doi.org/10.1051/swsc/ 20 12022.

Jin, S.G., J. Wang and P.H. Park (2005). An improvement of GPS height estimates: Stochastic modeling, Earth Planets Space, 57 (4), 253-259; doi:10.1186/BF 03352561.

Jin, S.G., O.F. Luo and P.H. Park (2008). GPS observations of the ionospheric F2-layer behavior during the 20th November 2003 geomagnetic storm over South Korea, J. Geodesy, 82 (12), 883-892; doi:10.10 07 / s00190-008-0217-x.

Leandro, R.F., R.B. Langley and M.C. Santos (2008). UNB3m_pack: A neutral atmosphere delay package for GNSS, GPS Solut., 12, 65-70.

Lejeune, S., G. Wautelet and R. Warnant (2012). Ionospheric effects on relative positioning within a dense GPS network, GPS Solut., 16 (1), 105-116; http:/ / dx. doi.org/10.1007/s10291-011-0212-1.

Li, X., M. Ge, B. Guo, J. Wickert and H. Schuh (2013). Temporal point positioning approach for real-time GNSS seismology using a single receiver, Geophys. Res. Lett., 40, 5677-5682; doi:10.1002/2013GL057818.

Li, X., M. Ge, J. Dousa and J. Wickert (2014). Real-time precise point positioning regional augmentation for large GPS reference networks, GPS Solut., 18, 6171; doi:10.1007/s10291-013-0310-3.

Li, X., F. Zus, C. Lu, G. Dick, T. Ning, M. Ge, J. Wickert and H. Schuh (2015a). Retrieving of atmospheric parameters from multi-GNSS in real time: Validation with water vapor radiometer and numerical weather model, J. Geophys. Res. Atmos., 120, 71897204; doi:10.1002/2015JD023454.

Li, X., M. Ge, X. Dai, X. Ren, M. Fritsche, J. Wickert and H. Schuh (2015b). Accuracy and reliability of multi-GNSS real-time precise positioning: GPS, GLONASS, BeiDou, and Galileo, J. Geodesy; doi:10. 1007 / s00190-015-0802-8.

Liu, Z. (2011). A new automated cycle-slip detection and repair method for a single dual-frequency GPS receiver, J. Geodesy, 85, 171-183; doi:10.1007/s00190010-0426-y.

Ngwira, C.M., G.K. Seemala and J.B. Habarulema (2013). Simultaneous observations of ionospheric irregularities in the African low-latitude region, J. Atmos. Sol.-Terr. Phy., 97, 50-57; http: / / dx.doi.org/10.10 $16 /$ j.jastp.2013.02.014.

Odijk, D. (2000). Improving Ambiguity Resolution by Applying Ionosphere Corrections from a Permanent GPS Array, Earth Planets Space, 10 (52), 675-680.

Odolinski, R., P.J.G Teunissen and D. Odijk (2015). Combined BDS, Galileo, QZSS, and GPS single frequency RTK, GPS Solut., 19, 151-163.

Oinats, A.V., V.I. Kurkin and N. Nishitani (2015). Statistical study of medium-scale traveling ionospheric disturbances using SuperDARN Hokkaido ground backscatter data for 2011, Earth, Planets Space, 67, 22; http: / / dx.doi.org/10.1186/s40623-015-0192-4.

Otsuka, Y., K. Suzuki, S. Nakagawa, M. Nishioka, K. Shiokawa and T. Tsugawa (2013). GPS observations of medium-scale traveling ionospheric disturbances over Europe, Annales Geophysicae, 31, 163-172; http: / / dx.doi.org/ 10.5194/ angeo-31-163-2013.

Paziewski, J. and P. Wielgosz (2014). Assessment of GPS + Galileo and multi-frequency Galileo singleepoch precise positioning with network corrections, GPS Solut., 18, 571-579; http:/ / dx.doi.org/10.1007/ s10291-013-0355-3.

Paziewski, J. (2015a). Precise GNSS single epoch positioning with multiple receiver configuration for mediumlength baselines: methodology and performance analysis, Meas. Sci. Technol., 26 (3), 035002; http: / dx. doi.org/10.1088/0957-0233/26/3/035002.

Paziewski, J. (2015b). Study on desirable ionospheric corrections accuracy for network-RTK positioning and its impact on time-to-fix and probability of successful single-epoch ambiguity resolution, Adv. Space Res., online first; doi:10.1016/j.asr.2015.12.024.

Paziewski, J., R. Sieradzki and P. Wielgosz (2015). Selected properties of GPS and Galileo-IOV receiver intersystem biases in multi-GNSS data processing, Meas. Sci. Technol., 26 (9), 095008; http: / / dx.doi.org/ 10.1088/0957-0233/26/9/095008.

Prikryl, P., P.T. Jayachandran, S.C. Mushini and I.G. Richardson, (2014). High-Latitude GPS Phase Scintillation and Cycle Slips during High Speed Solar Wind Streams and Interplanetary Coronal Mass Ejections: A Superposed Epoch Analysis, Earth Planets Space, 66, 1-10; doi:10.1186/1880-5981-66-62.

Shiokawa, K., Y. Otsuka, T. Tsugawa, T. Ogawa, A. Saito, K. Ohshima, M.Kubota, T. Maruyama, T. Nakamura, M. Yamamoto and P. Wilkinson (2005). Ge- 
omagnetic conjugate observation of nighttime medium- and large-scale traveling ionospheric disturbances: FRONT3 campaign, J. Geophys. Res., 110, A05303; http:/ / dx.doi.org/10.1029/2004JA010845.

Sieradzki, R. (2015). An analysis of selected aspects of irregularities oval monitoring using GNSS observations, J. Atmos. Sol.-Terr. Phy., 129, 87-98; http:/ / dx.doi.org/10.1016/j.jastp.2015.04.017.

Sieradzki, R., and J. Paziewski (2015). Study on reliable GNSS positioning with intense TEC fluctuations at high latitudes, GPS Solut., online first; doi:10.1007/ s10291-015-0466-0.

Spencer, P.S.J., and C.N. Mitchell (2007). Imaging of fast moving electron-density structures in the polar cap, Annals of Geophysics, 50 (3), 427-434.

Teunissen, P.J.G. (1997). The geometry-free GPS ambiguity search space with a weighted ionosphere, J. Geodesy, 71, 370-38; http: / / dx.doi.org/10.1007/s00 1900050105.

Wanninger, L. (2004). Ionospheric disturbance indices for RTK and network RTK positioning, Institute of Navigation, ION-GNSS 2004 Proceedings, LongBeach, CA, USA.

Wautelet, G., and R. Warnant (2014). Climatological study of ionospheric irregularities over the European mid-latitude sector with GPS, J. Geodesy, 88, 223240; http:/ / dx.doi.org/10.1007/s00190-013-0678-4.

Wielgosz, P., I. Kashani, D.A. Grejner-Brzezinska (2005). Analysis of long-range network RTK during severe ionospheric storm, J. Geodesy, 79 (9), 524531; doi:10.1007/ s00190-005-0003-y.

Wielgosz, P., A. Krankowski, R. Sieradzki and D.A. Grejner-Brzezi ska (2008). Application of Predictive Regional Ionosphere Model to Medium Range RTK Positioning, Acta Geophys., 56, 1147-1161; http:/ / dx.doi.org/10.2478/s11600-008-0059-1.

Wilgan, K., W. Rohm and J. Bosy (2015). Multi-observation meteorological and GNSS data comparison with numerical weather prediction model, Atmos. Res., 156, 29-42; doi:10.1016/j.atmosres.2014.12.011.

$\mathrm{Xu}, \mathrm{G}$. (2007). GPS: theory, algorithms and applications, 2nd ed., Springer, Berlin.

Zumberge, J.F., M.B. Heflin, D.C. Jefferson, M.M. Watkins and F.H. Webb (1997). Precise point positioning for the efficient and robust analysis of GPS data from large networks, J. Geophys. Res., 102 (B3), 5005-5017.

${ }^{\star}$ Corresponding author: Rafał Sieradzki,

University of Warmia and Mazury in Olsztyn, Poland;

email: rafal.sieradzki@uwm.edu.pl.

C) 2015 by the Istituto Nazionale di Geofisica e Vulcanologia. All

rights reserved. 